\title{
A systematic review in select countries of the role of the pharmacist in consultations and sales of non-prescription medicines in community pharmacy
}

\author{
Linda van Eikenhorst MSc, Nde-Eshimuni Salema, PhD , Claire Anderson, Ph.D.* \\ Claire.anderson@nottingham.ac.uk
}

School of Pharmacy, University of Nottingham, University Park, Nottingham NG7 2RD, UK

*Corresponding author

\section{ABSTRACT}

\section{Background}

Much has been studied in regard to non-prescription medicines (NPMs), but the impact of greater emphasis toward patient self-selection of such agents is still not well understood, and evidence in the literature might be equivocal.

\section{Objective}

The aim was to examine whether or not pharmacist interventions are important in the sale of NPMs and to summarize the evidence of pharmacists' contribution in maintaining patient safety and improving the quality of consultations involving NPMs.

\section{Methods}

Seven online databases were searched to identify the literature on studies conducted within the UK and in countries comparable to the UK reporting on consultations and selling of NPMs published between 1980 and 2013. All study designs except for quantitative surveys were eligible for inclusion into the review. The data extraction and quality assessment were performed according to the National Institute for Health and Care Excellence guidelines. The data extracted from the studies were analyzed and presented qualitatively.

\section{Results}

Eighty-three studies from an original 12,879 citations were included in this review. Just under half of the studies were published between 2000 and 2009 ( $n=38 ; 46 \%)$. Thirty-three $(44 \%)$ of the studies were conducted in the UK. The review showed that in terms of the contribution of community pharmacy staff in consultations for NPMs, non-pharmacist staff dealt with a large proportion of the consultations and pharmacists were usually involved in the consultation through referral from non-pharmacist staff member. Counseling was not consistently offered to everyone. Where counseling was provided it was not always of sufficient quality. Consultations were performed much better when symptoms were presented compared to when people made a direct product request. Pharmacists were reported to conduct better consultations than non-pharmacist staff. There was evidence to suggest that where counseling was appropriately provided this afforded the person a safe environment to utilize their NPMs. 


\section{Conclusions}

Seeking methods to develop better engagement with customers accessing pharmacy services for NPMs is necessary to enhance the interaction between these two parties. Efforts to enhance the community pharmacy environment to bring about a more positive experience for people using pharmacy is needed at present and will be important if the model for the selection of NPMs is modified in the UK. More studies are needed to allow a better understanding of the impact self-selection may have on patient safety in the community pharmacy context.

Keywords: Non prescription medicines; Community pharmacy; Pharmacist; Pharmacy staff; Sale; Supply; Systematic review; consultations; Patient safety

\section{INTRODUCTION / BACKGROUND}

The law surrounding supply of non-prescription medicines (NPMs) varies from country to country. In Australia and New Zealand there is a pharmacist category of NPMs (S3) that can only be supplied with the intervention of and counseling from a pharmacist. In the US, NPMs are freely available for self-selection on open shelves and in large quantities. In much of Europe medicines may only be sold from a pharmacy. Finland all counseling about NPMs must be given by a pharmacist. Under the current United Kingdom (UK) law, there are two categories of NPMs: General sales list medicines and pharmacy medicines $(P)$, where supply of $P$ medicines is only permissible only under supervision of a pharmacist. Various stakeholders, including the General Pharmaceutical Council (GPhC) are examining a proposal to permit self-selection of $\mathrm{P}$ medicines in UK community pharmacies. One key feature this decision hinges on is the assurance that patient safety will not be compromised by this proposed change which is likely to result in a large number of currently available $P$ medicines being sold without the opportunity for a pharmacist to intervene. To gain an understanding of the possible impact this wider availability of $P$ medicines may have in UK community pharmacies, a review of studies examining the current role that pharmacists play when undertaking consultations of NPMs was undertaken. The overarching aim of this review was to examine whether or not pharmacist interventions are important in the sale of NPMs and to summarize the evidence of pharmacists' contribution in maintaining patient safety and improving the quality of consultations involving NPMs. Implicit in these aims is the need to examine non-pharmacist staff roles in supply of NPMs. A secondary aim of this review was to identify the future research agenda.

The sale and supply of medicines in the United Kingdom (UK) is regulated by the Medicines Act $1986 .{ }^{1}$ This act defines three medicine categories each with their own restrictions regarding the sale and supply of these medicines. Prescription-only medicines (POMs) can only be obtained from a pharmacy or a dispensing general practice surgery with a legal prescription written by a general practitioner or other 
suitably qualified health care professional. ${ }^{2}$ Pharmacy $(P)$ medicines are available without prescription but may only be sold from a registered pharmacy premise and the sale should be supervised by a pharmacist. The last group is the medicines on the general sales list (GSL). GSL medicines can be bought without a prescription and are available in any retail outlet. ${ }^{1}$ Most of the new medicines entering the market start as POM, but after a few years a medicine may be reclassified (deregulated). Reclassification is normally requested by the company that holds the marketing authorization, but could also be initiated by other interested parties, for example, the professional body or community pharmacy chains. All applications concerning reclassification are evaluated by the Medicines and Health Products Regulatory Agency (MHRA). They investigate whether or not a medicine could be reclassified according to several criteria included in the Human Medicines Regulations 2012, regulation 62 (3) (POM to P) and regulation 62 (5) (P to GSL). ${ }^{3}$

Various stakeholders, including the General Pharmaceutical Council (GPhC), are examining a proposal to allow self-selection of $\mathrm{P}$ medicines in UK pharmacies, without the supervision of a pharmacist. ${ }^{4}$ This move is being deemed necessary to allow patients better/easier access to medicines for the management of minor ailments. One key feature this decision hinges on is the assurance that patient safety will not be compromised by this proposed change. ${ }^{5}$

The primary aim of this systematic review was to identify and summarize the available evidence of the role pharmacists play in maintaining and guaranteeing patient safety and improving the quality of consultations for supply of $\mathrm{P}$ medicines, and to establish whether or not the intervention of a pharmacist is important in the sale of $P$ medicines in community pharmacies. A secondary aim was to proffer a research agenda in this area of pharmacy practice.

\section{Methods}

\section{Review team and review method}

This systematic review is reported in accordance with the Preferred Reporting Items for Systematic Reviews and Meta-Analyses (PRISMA) statement. ${ }^{6}$

The review team consisted of the main reviewer $L v E$, NS and CA. The systematic review was undertaken based on the five-step principle of conducting a systematic review as described by Khan et al, which includes defining the research question, identifying the literature, quality assessment of the selected literature, summarizing the findings and interpreting the evidence. ${ }^{7}$ In this review, the question was defined by the Royal Pharmaceutical Society, and hence the authors entered the review at step two of the process.

\section{Literature identification}

\section{Databases searched and search strategy}


A multiple database search spanning 33 years (1980-2013) was performed to identify relevant literature for inclusion in the review in June 2013. Databases searched included Embase, International Pharmaceutical Abstracts (IPA) and Psyclnfo (all searched via OVID), SCOPUS, Web of Science, Cochrane, Applied Social Sciences Index and Abstracts (ASSIA) and Medline.

A list of Medical Subject Headings (MeSH) terms, MeSH-like terms and free text words with wild card truncations were combined to develop search strategies to capture the community pharmacy setting, NPM sales and condition for which NPMs are currently used. The exact terms used to run the search were tailored for the requirements of the individual databases (see Table 1). Searches were restricted to studies published either in English or in Dutch.

\section{Criteria for including studies in the review}

Included studies were conducted in the UK, Ireland, Australia and New Zealand, which have a similar community pharmacy context to the UK. Studies further afield from other European countries and North America were scoped to gain insight into the realities to be considered if NPMs are made available for self-selection. All titles and abstracts for articles retrieved were screened for relevance against the inclusion/exclusion criteria listed in Table 2 by the main reviewer (LVE). A second reviewer (NS) screened $10 \%$ of these to verify the selection of studies. Full articles of titles and abstracts retained at this stage were obtained for further reading. In the second round of the review the main reviewer (LVE) reviewed all the articles for relevance whilst the other two reviewers (CA and NS) reviewed two-thirds of the papers each, with one-third articles overlapping. During this second round of the review the main reviewer (LVE) also searched the bibliographies of the retrieved articles for additional relevant articles. At all stages of the review disagreements between reviewers were resolved by discussion. 


\begin{tabular}{|c|c|c|c|}
\hline & Database MeSH term & Map Term/ Subject Heading & MeSH term \\
\hline Keyword & Medline/PubMed & Embase & Cochrane \\
\hline Pharmacist(s) & Pharmacists & Pharmacist & Pharmacists \\
\hline Pharmacy/ies & Pharmacies & Pharmacy & Pharmacies \\
\hline Community pharmacy/ies & Community Pharmacy Services & $\begin{array}{l}\text { Pharmacy (community pharmacy } \\
\text { services is a specific term within } \\
\text { pharmacy) }\end{array}$ & Community Pharmacy Services \\
\hline Non prescription & Nonprescription drugs & non prescription drug & Nonprescription drugs \\
\hline Non prescripton medicine(s) & Nonprescription drugs & non prescription drug & Nonprescription drugs \\
\hline отC & Nonprescription drugs & non prescription drug & Nonprescription drugs \\
\hline over the counter & Nonprescription drugs & non prescription drug & Nonprescription drugs \\
\hline OTC medicine(s) & Nonprescription drugs & non prescription drug & Nonprescription drugs \\
\hline over the counter medicine(s) & Nonprescription drugs & non prescription drug & Nonprescription drugs \\
\hline OTC drug(s) & Nonprescription drugs & non prescription drug & Nonprescription drugs \\
\hline over the counter drug(s) & Nonprescription drugs & non prescription drug & Nonprescription drugs \\
\hline Pharmacist only drug(s) & Behind-the-Counter drugs & Not found & Behind-the-Counter drugs \\
\hline P-meds & Behind-the-Counter drugs & Not found & Behind-the-Counter drugs \\
\hline P-medicine(s) & Behind-the-Counter drugs & Not found & Behind-the-Counter drugs \\
\hline Pharmacy meds & Behind-the-Counter drugs & Not found & Behind-the-Counter drugs \\
\hline
\end{tabular}




\begin{tabular}{|l|l|l|l|} 
Self care & $\begin{array}{l}\text { Behind-the-Counter drugs } \\
\text { Self Care (Very broad, includes also } \\
\text { blood glucose self monitoring, self } \\
\text { administration and self medication) }\end{array}$ & Self care & Behind-the-Counter drugs \\
Self care medication & Not found & Not found & Not found \\
Self care medicine(s) & Self Medication & Self medication & $\begin{array}{l}\text { Slood glucose self monitoring, self } \\
\text { administration and self medication) }\end{array}$ \\
Self medication & Not found & Not found & Not found \\
Minor ailment(s) & Not found & Not found & Self Medication \\
Minor illness & Not found & Not found
\end{tabular}

Table 1: Search terms used to identify articles for review 


\section{Data extraction}

Data extraction was undertaken by the main reviewer (LVE) and by a second reviewer (NS). The data extracted by the main reviewer was then summarized according to the National Institute for Health and Clinical Excellence (NICE) methods. ${ }^{8}$ Three different data extraction forms were used to reflect the nature of the study designs adopted in the studies being reviewed, namely qualitative, quantitative and mixed methods study designs.

Questionnaires were included only if they we part of a larger study. The data extraction form for the studies with a mixed method design was constructed by combining important points from the qualitative and quantitative tools. The suitability of the templates was confirmed by the main reviewer (LVE) running a test data extraction exercise on each of the study designs in discussion with the other two reviewers.

\section{Quality assessment}

The quality assessment of the papers included in the review was performed by the main reviewer with guidance from the remaining two reviewers. The quality assessment checklists used were also developed from the NICE method mentioned above. To provide a method of comparing quality for the different study designs percentages of adhering with checklist was computed. Every item of the checklist was judged and could be answered either positively or negatively/unclear. A percentage was calculated out of the number of positively answered questions to the total number of questions. The quality ranking was as following; low = less than 33\% of the items was positively answered, medium $=33-66 \%$ and high over $66 \%$ of the questions was answered positively (see Table 3.)

\section{Number of studies included in the review}

A total of 12,779 citations were obtained from the database searches. After the initial reading titles and abstracts 570 studies were identified as being relevant for the review. Full papers for these studies were obtained for further reading. A total of 83 papers, representing 76 unique studies were retained for inclusion into the review (Fig. 1). 


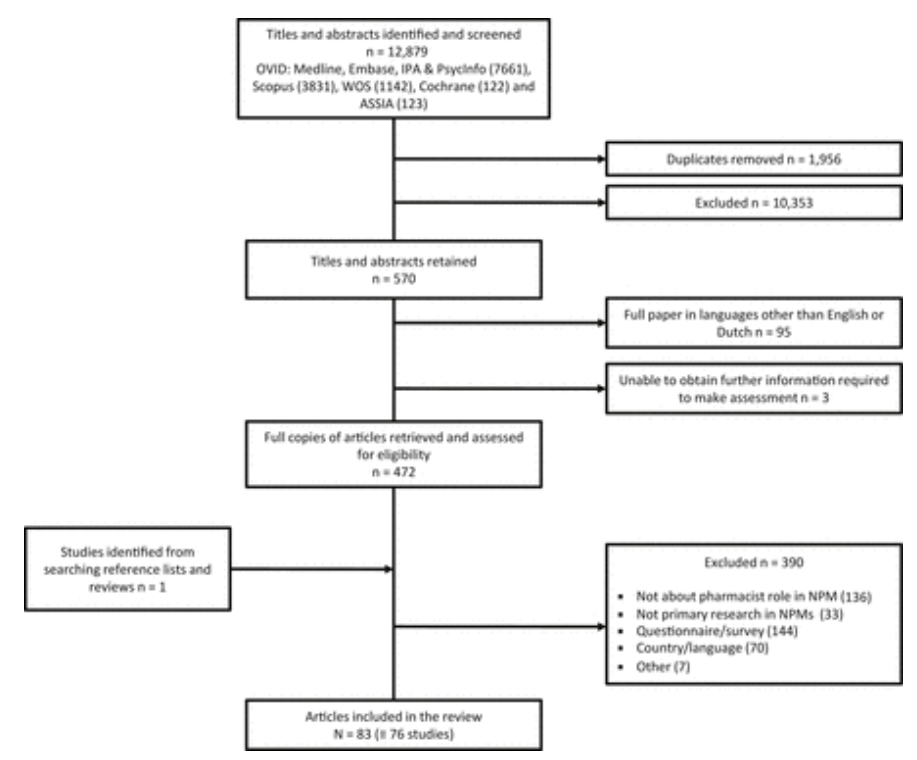

Fig 1 Identification of studies for review

The main features of studies included in the review have been presented in Table 3.

\section{General study characteristics}

Table 3 shows that the availability of studies to review increased with each decade with the number of available studies to review in the last decade (2000-2010) $(n=39 ; 47 \%)$ nearly double those available in the nineties $(n=20 ; 24 \%)$ and nearly eight times more than those available in the eighties $(n=5 ; 6 \%)$.

The majority of the studies reviewed were conducted in Europe ( $n=54 / 83 ; 65 \%)$ with the UK and Ireland representing the largest proportion of these $(n=38 / 83 ; 46 \%)$. The second largest set of studies originated from Australasia ( $n=20 / 83 ; 24 \%$ ) while North America accounted for approximately $10 \%(n=8)$ of the studies reviewed.

In the majority of studies the use of theoretical framework was not mentioned or reason for absence stated ( $n=67 / 83 ; 81 \%$ ). A number of different theories featured in the studies reviewed; a few couple of studies were based on the same theoretical underpinning.

\section{Features of consultations for NPMs}

Consistent with the aims of the study, the roles of other staff in addition to pharmacists were examined. In many of the studies it was difficult to distinguish which of the pharmacy staff had been involved in supply of NPMs. The involvement of pharmacists and pharmacy staff in consultations of NPM included four key aspects namely: questioning and information gathering, product recommendation and sale, provision of information and advice, and referrals (Fig. 2). The results here attempt not only to describe these activities that are undertaken during a consultation for NPMs but also describe whether these activities were carried out effectively. 


\begin{tabular}{|c|c|c|}
\hline Criterion & Category & No of studies and reference \\
\hline \multirow[t]{4}{*}{ Year } & $1980-1989$ & $5 \quad(9,10,11,12,13)$ \\
\hline & $1990-1999$ & $20(14,15,16,17,18,19,20,21,22,23,24,25,26,27,28,29,30,31,32,33)$ \\
\hline & $2000-2009$ & $\begin{array}{l}38(34,35,36,37,38,39,40,41,42,43,44,45,47,48,51,52,54,56,57,58,59,60,61 \\
62,63,64,65,66,67,68,69,70,71,77,81)\end{array}$ \\
\hline & $2010-2013$ & $20(15,57,58,73,74,76,77,81,78,79,80,81,82,83,84,85,86,90,91)$ \\
\hline \multirow[t]{8}{*}{ Countries } & Australasia & \\
\hline & Australia & $18(12,37,38,41,42,48,50,52,53,55,62,80,82,86,87,89,90,91$ \\
\hline & New Zealand & $2(54,70)$ \\
\hline & North America & \\
\hline & Canada & $3(51,32,33)$ \\
\hline & USA & $5(14,22,28,56,60)$ \\
\hline & Germany & $4(34,39,76,79)$ \\
\hline & Ireland & $1(19)$ \\
\hline
\end{tabular}




\begin{tabular}{|c|c|c|}
\hline & UK & $\begin{array}{l}37(10,11,13,15,16,17,18,20,21,25,26,27,28,29,31,35,43,5547,57,59,61,63,65.66, \\
67,68,72,75,77,78,81,83,84,88\end{array}$ \\
\hline & The Nordic countries & \\
\hline & Denmark & $1(85)$ \\
\hline & Finland & $3(23,49,58)$ \\
\hline & Norway & $1(44)$ \\
\hline & Sweden & $6(24,26,69,70,71,74)$ \\
\hline & The Netherlands & 1 (9) \\
\hline & Transnational (Australia, Germany and UK) & $1\{40)$ \\
\hline \multirow[t]{6}{*}{$\begin{array}{l}\text { Data collection } \\
\text { methods }\end{array}$} & Focus groups & $3(46,49,60)$ \\
\hline & Interviews & $\begin{array}{l}32(9,10,11,18,20,21,25,35,41,42,46,48,51,52,56,57,62,67,69,70,73,, 77,78,80, \\
81,83,84,85,87,88,91)\end{array}$ \\
\hline & Audio-recording consultations & $4(16,17,18,29)$ \\
\hline & Observations & $17(9,16,17,19,20,21,26,30,32,33,36,41,42,47,49.61,63,65)$ \\
\hline & Mystery shopper & \\
\hline & with feedback)used as an intervention & $11(37,38,39,76,50,53,58.63,64,65,66,91)$ \\
\hline
\end{tabular}




\begin{tabular}{|c|c|c|}
\hline & used to report back on aspects of consultation & $10(14,22,28,34,74,12,44,54,59,86)$ \\
\hline & $\begin{array}{l}\text { Audit type recording of activities related to consultations for NPMs or use of } \\
\text { community pharmacies }\end{array}$ & $16(13,15,24,31,43,45,46,68,69,70,71,72,75,77,81,82,90)$ \\
\hline & Health diaries and other records made by patients & $5(10,11,47,55,89)$ \\
\hline & Surveys & $7(40,55,70,77,81,89,71)$ \\
\hline & Critique of video-vignettes & $1(23)$ \\
\hline \multicolumn{3}{|l|}{$\begin{array}{l}\text { General study } \\
\text { themes }\end{array}$} \\
\hline & Observing / examining aspects of consultations or use of NPMs & $\begin{array}{l}51(9,12,13,14,16,17,18,19,20,21,22,23,24,29,30,31,32,33,34,36,37,38,39,41,42,47,49.51,53,54, \\
59,60,61,63,64,65,66,68,72,74,76,77,79,81,82,86,90,91)\end{array}$ \\
\hline & Recording 'auditing' aspects of consultations & $2(43,45,68)$ \\
\hline & Work sampling & $3(26,15,75)$ \\
\hline & Exploring experiences and perceptions of & \\
\hline & Pharmacists & $8(48,49,57,6167,83,84,88)$ \\
\hline
\end{tabular}




\begin{tabular}{|c|c|c|}
\hline & Pharmacy staff (pharmacists and non-pharmacists) & $2(78,91)$ \\
\hline & GPs & $1(65)$ \\
\hline & Customers & $18(9,18,20,21,25,27,41,42,47,52,56,60,62,73,80,85,87)$ \\
\hline & Customers as 'representatives' & $4(10,11,77,81)$ \\
\hline & Equipping / supporting customers to manage minor ailments & $2(55,89)$ \\
\hline & $\begin{array}{l}\text { Upskilling pharmacy teams to provide better customer care / change to practice } \\
\text { to improve customer care }\end{array}$ & $4\{35,40,70,71)$ \\
\hline & Increasing access to care & $1(46)$ \\
\hline \multirow[t]{3}{*}{$\begin{array}{l}\text { Participants/those } \\
\text { involved }\end{array}$} & Pharmacists & $\begin{array}{l}28(12,14,5,18,22,26,28,29,30,40,43,48,49,53,57,58,59,58,61,69,75,76,77,79,81, \\
83,84,86 .)\end{array}$ \\
\hline & Pharmacy staff (pharmacists and non-pharmacists) & $\begin{array}{l}35(9,16,17,19,20,21,24,31,32,33,34,35,36,37,38,39,41,42,44,47,50,54,63,64,65,66,68,70,71, \\
72,74,78,89,90,91)\end{array}$ \\
\hline & GPs & $2(40,57)$ \\
\hline
\end{tabular}




\begin{tabular}{|c|c|c|}
\hline & Customers & $26(9,13,18,20,21,27,41,42,43,46,47,51,52,54,56,60,61,62,68,70,73,80,82,85,87,89)$ \\
\hline & Customers as 'representatives' & $5(77,81,10,11,58)$ \\
\hline \multirow{9}{*}{$\begin{array}{l}\text { Theoretical } \\
\text { framework }\end{array}$} & Agenda-led outcome based analysis (ALOBA) & 1 (66) \\
\hline & Cognitive psychology perspective & $1(23)$ \\
\hline & Education outreach based on IDEALS & $2(63,65)$ \\
\hline & Grounded theory & $2(20,21)$ \\
\hline & Human error theory & $2(63,65)$ \\
\hline & Motivational interviewing & $2(53,91)$ \\
\hline & Pharmacy practice risk management framework & $1(48)$ \\
\hline & Self-management theory & $1(89)$ \\
\hline & Self-regulation of illness behaviour & $1\{55)$ \\
\hline
\end{tabular}




\begin{tabular}{|l|l|l} 
& Theory of planned behaviour & $2(56,67)$ \\
\hline & Transtheoretical framework of change & $2(43,68)$ \\
\hline Quality Score & HIGH ++ & $53(14,15,16,17,18,20,22,24,34,35,37,39,41,42,44,45,48,49,50,51,52,54,55,56,57,58$, \\
& & $59,60,61,62,63,64,65,66,67,68,70,71,72,73,76,77,78,79,80,81,83,84,85,86,87,89,90,91)$ \\
& MEDIUM+ & $23(9,10,11,13,19,21,23,25,26,27,29,31,32,36,40,43,53,69,74,75,76,82,88)$ \\
& & $6(12,28,30,33,38,46)$ \\
& LOW - & $1(47)$ \\
& & \\
\hline & Not possible TO SCORE & \\
& &
\end{tabular}

Table 3 Characteristics of studies 


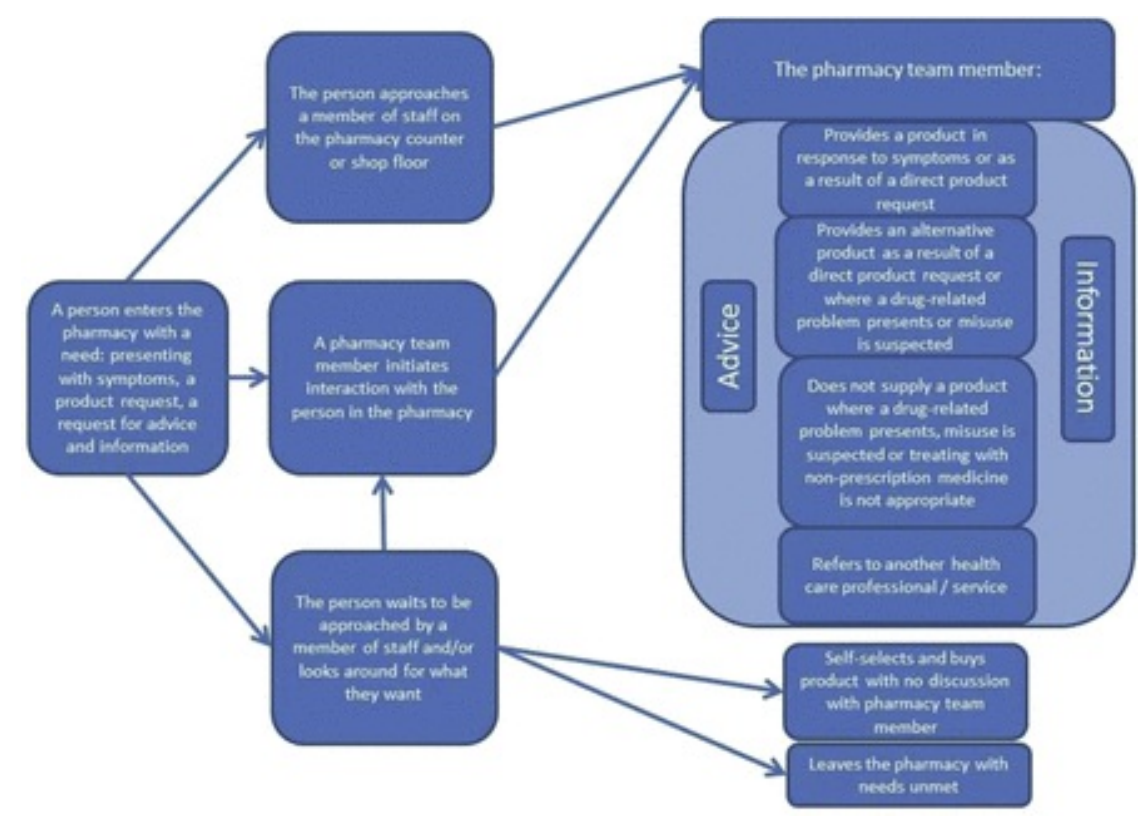

Fig. 2 Schematic of a person's visit to the pharmacy and the roles undertaken by the pharmacy team. Questioning and information gathering from customers

A number of studies included in the review reported on pharmacy staff questioning and gathering information from people during a consultation for an NPM before a differential diagnosis could be pronounced and subsequent action in response to this diagnosis undertaken.

Approaches taken by the different studies reviewed to capture this information varied widely and included reporting on number of questions or which questions were asked or proportion of consultations where questions were asked $^{12,14,29,34,39,50,54,59,66,74,76,77,86}$; reporting of the appropriateness of the questions asked or questioning style 16,59 ; reporting on the appropriateness of patient assessment $20,22,23,40,58,65$; reporting on change in use of questioning behavior following an intervention 53,66; and reporting on consumers' experience and expectation of questioning by pharmacy staff. 25,85

Because this feature of the consultation was measured using different measures it is not 
possible to provide a single overall outcome of how questioning was performed, but in general terms the studies indicated that adequate questioning and information gathering was not carried out fully or consistently during consultations. Pharmacy staff were seen as lacking the necessary questioning skills, listening skills and knowledge to undertake adequate information collection 65 and advice provision as a reason for not questioning customers properly. On the other hand, assuming customers' knowledge of products as a result of previous purchase was also reported as a reason why questioning was not done effectively. ${ }^{77}$ Criticism was directed toward pharmacy staff in their use of mnemonics; this was described as sometimes being 'wooden or unresponsive.' 16

Pharmacy staff performed better when they were responding to symptoms compared to when they were approached by a patient with a direct product request. This included attaining higher overall scores when responding to symptoms in terms of questioning 74 ; undertaking the process of questioning more effectively, ${ }^{54}$ and achieving higher levels of appropriate patient assessment when responding to symptoms. ${ }^{22}$ There was an indication from some studies reviewed that in reality pharmacy staff are faced with more product demands from customers rather than being presented with symptoms. 20,72 It was suggested therefore that in practice, pharmacy staff are in fact likely to be dealing with customers who know what they want and may account for the reported hostility and negative customer response directed at pharmacy staff when attempting to question customers 20,65 On the other hand the negative manner in which customers responded to questioning by pharmacy assistants rose suspicion amongst staff that a customer was in fact misusing a product. 78

Telephone interviews revealed that when it came to unsolicited questioning, there was different expectation of being questioned, awareness of why questions were asked or the degree to which the person was responsible to volunteer information to the pharmacy staff even if it had not been requested. ${ }^{25}$ Furthermore, people mentioned the challenges associated with attempting to answer questions on behalf of another person for whom they were purchasing medicines. Challenges included not having sufficient information about the other person the pharmacy staff were enquiring about in order to provide comprehensive answers to questions and sharing confidential medical problems of the third party with pharmacy staff.

The use of additional support aids during the consultation such as the use of pharmacy decision support system (PDSS) showed that this resulted in community pharmacists covering a wider range of mandatory questions to confirm appropriateness of selfmedication. 76

The studies also revealed that there was scope to improve the pharmacy staff's questioning behavior through the use of interventions. A study that used mystery shopper with feedback reported an improvement in the use of open-ended questions from a

baseline score of $0.03 \%-39.5 \%$ as this intervention significantly improved this aspect in the intervention group from $0.03 \%$ to $68 \% .87$ 
Through the process of questioning and information gathering pharmacy staff were able to identify and deal with drug related problems; in some instances drug issues were missed by pharmacy staff. ${ }^{34}$ Example of drug related problems picked up included inappropriate selfmedication, 51,79 requested product inappropriate, 72,79 intended duration of drug use to high, ${ }^{79}$ wrong dosage, ${ }^{79}$ unsure of indication of medication, ${ }^{69}$ or therapy failure. ${ }^{69}$ One study reported identifying at least one drug-related problem in $19.5 \%$ of product requests and in $12.5 \%$ of symptom presentation requests. ${ }^{79}$ This same study showed that using a person's medical history when checking the suitability of use of NPMs resulted in issues such as wrong dosage and drug-drug interactions to be picked up more readily. ${ }^{79}$ In some instances patients were the ones who picked up the drug related problems and relayed these to the pharmacy staff $36,69,70$ to deal with. A study that audited the number of drug related problems identified depending on whether sales of NPMs in pharmacies occurred over the counter or through a self-service area revealed that on average the identification of drug related problems was $60 \%$ higher in the pharmacies selling NPMs over the counter. 69

Some pharmacists were commended for helping people to seek help for their misuse through questioning the frequent users of codeine. 87 Both pharmacists 78 and patients 87 viewed interactions regarding dealing with product misuse as a negative experience.

\section{Product recommendation and sale}

Product recommendation or sale of an NPM following response to symptoms or a direct product request was a common feature of NPM consultations studied in this review. As the conditions for which the NPMs were recommended and sold varied widely, so did the classes of medicines supplied. Product recommendation in the pharmacies was usually for self-use, however obtaining NPMs for another person, especially children, was also a feature observed in the studies reviewed.

According to one study, many people (57\%) considering purchasing a medicine for a first time identified pharmacy staff as the single most important influence in helping them make this decision. ${ }^{41}$ Advertisements of medicines both in pharmacies or through the media accounted for about $6 \%$ of this influence.

The use of evidence-based medicine on the part of the pharmacist for choice of product to recommended was not widely reported, where this was studied it appeared that the use of evidence by pharmacists in product choice was a secondary consideration. The main concern of these pharmacists studied was satisfying themselves of lack of potential harm to the customer; their primary concern was patient safety. $67,83,84$ In spite of this concern for safety, studies revealed that in some cases consultation included selling of products where drug interactions have been missed or not discussed with the customer, ${ }^{34}$ inappropriate product recommendations $12,14,22,39,59,66,74,76$ and in some cases no product being recommended altogether was evident. ${ }^{74}$ Moreover, there were reports from pharmacy staff that they would partake in selling of inappropriate or unnecessary products because of the pressure they felt from some people. 83 
There was a higher number of people who came into the pharmacy with a brand of medicine to purchase in mind compared to those who did not. ${ }^{41,42}$

Some positive involvement of pharmacy staff had in product recommendation included helping people to find the medicine on the shelf or in offering people alternative products to what they had in mind or had previously tried. People who had walked into the pharmacy with a particular brand in mind were in some instances successfully offered cheaper products. ${ }^{41}$ It was noted however, that in some cases where the brand was a sure preference a cheaper substitute would not usually be accepted. ${ }^{81}$ Where someone presented with a drug-related problem one option for pharmacy staff was to recommend a different product. Some people viewed their experience in a negative light if they were expected to be responsible for making the decision on what product to purchase, for these people the pharmacist was deemed to have failed them in terms of providing them with direction. 61

Recommending an alternative product was a common intervention undertaken by pharmacy staff in response to identification of a drug related problem. $69,70,72,79$ Pharmacy staffs' monitoring and surveillance of sales was a key way in which potential abusers of medicines were identified. $43,68,78$ In fact, for both pharmacist and pharmacy staff (but mainly staff) the frequency of purchase was central for defining and identifying suspected OTC misuse or abuse. ${ }^{78}$ For pharmacy staff factors such as frequency (been in two or three times; buying the same thing); product knowledge (know exactly where a product is kept; know exact product size); large quantities being purchased/requested; manner in which customers respond to questions; negative reaction or aggression to a refused or referred sale; use of lies and deception to attain a product (pretending to buy for another person); use of others to purchase medicines for them raises suspicion to the fact that there may be likely product misuse. ${ }^{78}$ Pharmacists taking part in an intervention that involved identifying instances of potential abuse and misuse provided a list of features they suggested could discriminate between the two behaviors. ${ }^{68}$ These features for misuse include willingness to converse with pharmacists to seek information and were amenable to advice given or refusal of product. On the contrary, abuse was likely where products were purchased more frequently, negative reaction to a refused sale, justification for purchase of product, those who visited the pharmacy in a particular pattern. 68

Pharmacy staff placed emphasis on using regulatory guidelines and formal pharmacy procedure for instance referring to pack size or indication changes for products; recording sales of medicines of potential abuse in the pharmacy; use of technology able to control and monitor sales as a means of managing suspected abuse. ${ }^{78}$ Refusing a sale was one way pharmacists dealt with the problem of drug misuse and abuse. ${ }^{78}$

An educational intervention to support the implementation of evidence based guidelines in $\mathrm{CP}$ setting for the treatment of vulvovaginal candidiasis showed that there was no difference found between groups when reporting on the proportion of visits resulting in an 
appropriate sale or non-sale of the anti-fungal product as per guidelines. ${ }^{64}$ This appropriate outcome was found to be highly significantly positively associated with the total information score and WWHAM score. ${ }^{63}$

A study that evaluated the impact of pharmacy staff in terms on clinical interventions related to pharmacy and pharmacy only medicines showed that these interventions averted emergency medical attention or serious harm and could be classed as potentially life-saving. As a result of these interventions urgent GP visits were shown to have been avoided and so were admissions to, and emergency departments, standard wards and intensive care. Additionally, exacerbations of an existing condition and adverse drug effects were also avoided. 90

\section{Providing information and advice to customers}

Many studies reported on aspects of provision of advice and information to customers during pharmacy consultations. Similar to questioning and information gathering, many different aspects provided the focus to determine whether pharmacy staff carried out this role effectively.

While pharmacists were identified as the predominant source of information for NPMs. 62 There was a large number of instances where no advice and/or information provision was recorded. One study demonstrated the disparity of advice giving between pharmacies by noting that when comparing pharmacies, in only five pharmacies (50\% of pharmacies) did the percentage of people who received advice (72\%) was shown to be greater than percentage that did not receive advice. 16

In some situations advice and information was provided if the person prompted the member of staff. ${ }^{33,39}$ One observation study noted people's patterns of behavior in that more of them looked at the product on the shelf before asking for advice. Other studies reported on provision of unsolicited advice and information 77 An interview study with people purchasing laxatives showed that it was customary for customers to search shelves and self-select products rather than engage with a pharmacy staff member; a behavior that was borne from being unsatisfied with level of assistance received in the past. ${ }^{52}$

Timing of when customers asked for advice was grouped into those who sought advice after they spent time looking at the product where the other group of consumers proceeded to seek advice before looking at the product. ${ }^{32}$ Difference in advice and information provision was identified in consultations that were rooted in responding to symptoms versus those that arose from a direct product request. Counseling was more likely to be given when someone presented their

symptoms or medical problems compared to situations where a product was requested by brand name or therapy group. 49

Where written information was given one study reported that two-thirds of people did not read the written information, those who read were mainly interested in administration and 
side-effects. ${ }^{9}$ With regards to the level of advice provided, most did not want more verbal advice (92\%) whereas only $8 \%$ mentioned they would have wanted more verbal advice. ${ }^{9}$ One study reported that people felt the need for different levels of advice depending on whether they had purchased a medicine before, with more advice and information thought to be needed when a medicine was new. ${ }^{77}$

Some medicine-related problems were dealt with by pharmacy staff through providing advice and information. This included provision of counseling on the offending medicine and its associated problems $69,70,79$ through provision of written material, ${ }^{79}$ or through provision of practical assistance with for instance devices. ${ }^{69,79}$ Although pharmacists were reported to provide information on the maximum dose of codeine, frequent users of codeine accused some pharmacists for their lack of a proactive approach in providing information on the risks of codeine but instead responded reactively once problems with misuse had deeply embedded. 87 Pharmacists engaged on activities such as drawing on evidence based medicine, their clinical knowledge and expertise which included advising customers when it came to deal with the subject of medicine abuse and misuse. ${ }^{78}$

A pilot ${ }^{55}$ that examined the impact of goal setting against provision of standard care for the management of intermittent allergic rhinitis showed that goal setting with health care professionals of the pharmacy team showed a significant reduction in symptom severity measured over the period of the study, with the patients in the health care professional group presenting with significantly lower mean perceived symptom severity scores compared to the standard care group. A full intervention to assess this intervention 89 revealed that there was a significant improvement in all outcome measures over time within each of the groups $(P<0.005)$, but no significant difference between control and intervention group over time. However allergic rhinitis self-efficacy, symptom severity, and quality of life improved significantly in patients assisted by pharmacy staff.

Another intervention study showed that advice given from a pharmacist lead to greater symptom relief by those people who reported following the advice. ${ }^{71}$ Further investigation to review those whose compliance with advice was classified as appropriate by the independent assessors, and who had claimed to have been completely compliant with advice, a higher number from this group experienced great relief of symptoms than those who only partially followed and reported the same level of improvement $(P<0.0001) .{ }^{71}$

An interview study 80 revealed that some people did in fact leave the community pharmacy with unanswered health related questions, some regarding NPMs. It was postulated that adverse events that may result because of this may include non- adherence, suboptimal treatment and adverse events (for all the unanswered health related queries not just the NPM ones). Past unanswered health-related questions served to highlight that the implications of having unanswered questions related to NPMs included the pharmacy losing their custom, anxiety over the use of medication, a delay in receiving effective treatment.

The age of the patient was mentioned as a factor that had an impact on provision of advice during consultations for NPMs. Younger people (aged 20-44 years old) were significantly 
more likely to receive advice when busying medicines than people aged over 65 years. ${ }^{19}$ It was also reported that providing advice when counseling older people at times provided challenging as sometimes older people were not clear on what their needs were and at times were not able to clearly communicate their symptoms. ${ }^{49}$ It was also mentioned that people from different cultures at times had problems communicating their symptoms which too made provision of advice a challenge. 49

An interesting aspect reported in one of the studies was the amount and type of information and advice that was recalled. ${ }^{18}$ This UK study showed that of the mean 13.74 items of information offered to someone by the pharmacist during a consultation, only a mean of $3.26(23.7 \%)$ was actually recalled. It was shown that procedural advice (36.9\%) was more likely to be recalled than non-procedural advice $(17.5 \%)(P<0.001)$ in this study. Furthermore, the study revealed that it was in fact information that was repeated more frequently that was subsequently recalled.

\section{Referrals}

Although referrals could arguably be seen as an extension of advice provision, the separation here is in recognition that within consultations for NPMs a number of different types or referrals took place, namely referral of a patient from pharmacy staff to a pharmacist within the community pharmacy setting, $16,17,48,72,78$ referral of patients from pharmacy staff to other health care professionals (usually GPs), $10,18,21,24,28,31,37,39,4549,53,54,59,67,69-72,78,79$ referral from other health care services to community pharmacy and self-referral to GPs by people via the community pharmacy route. $10,21,47$

In cases where pharmacy staff suspected that they were dealing with misuse cases they would refer the person to the pharmacists. ${ }^{78}$ Occasional signposting and involving the people's GP was a strategy used by pharmacists in dealing with medicine misuse and abuse. 78

Referring people to other health care professionals, mainly GPs was another way that community pharmacy staff dealt with other drug related problems identified during consultations for NPMs. ${ }^{69,72,79}$ One study did, however, illustrated that people did erroneously recall being referred to their GP by the pharmacist when in fact this was not the case. $^{18}$

Although pharmacists had referred people in response to refusing a supply there was evidence from the interviews that these too were not that straight forward due to lack of information about the person (and therefore not being able to get in touch with their doctor), not having in-depth knowledge of services that are available to support those abusing medicines and using a method of cascading information of someone suspected of abuse although used by some was not always practical due to the time and number logistics. ${ }^{78}$ 
A number of studies mentioned that some people undertook a visit to the community pharmacy as a 'stepping-stone'; this provided a legitimate path for them to then visit the GP, at times as a result of a referral from community pharmacy. ${ }^{10,11,21,47}$ There were some people, however, who did not have trust in NPMs, and for these customers selfreferral to GP was the chosen option to deal with a medical condition. 47

At times referrals were deemed to be inappropriate, ${ }^{21}$ with the 'play safe' attitude of pharmacists in situations of doubt and uncertainty accounting for some of these unnecessary referrals. With regards to appropriateness of referrals it was identified that there were instances when community pharmacy staff should have referred someone to the GP and failed to do so. ${ }^{39}$ In addition to this, the reasons why referrals did not make it to their GP is because people did not always follow advice from the pharmacy, even in situations where in follow-up consultations people had promised to visit their GP as per referral. 49,70 Sometimes advice from pharmacist to visit GP was not heeded as the customer felt that their symptoms did not warrant such a visit. ${ }^{18}$ Consequently, there was concern among community pharmacy staff that having consultations and times the provision of an NPM in a delayed referral in fact may serve as a barrier to going to the GP and had a potential of masking a serious medical problem. ${ }^{67}$ In fact, another study reported that $57 \%$ of people questioned would have taken their medical problem to the GP if a pharmacy service supplying NPMs was not available. It was recognized however that referral was not always easy, 49 and ultimately the person has to be willing to go to the GP. 70 Interventions to improve referrals showed that at times these did not have a significant impact $^{37}$ and at times interventions did increase the number of referrals. ${ }^{70}$

\section{Factors affecting consultations of NPMs}

\section{Pharmacists versus non-pharmacist involvement}

Reviewing studies that measured the involvement of pharmacists and non-pharmacist staff in consultations for NPMs showed that non-pharmacist staff dealt with more of the consultations than did pharmacists. $16,17,31,63$ Pharmacists were usually involved in these consultations by invitation from pharmacy staff as cases were referred to the pharmacist for their input. Pharmacists interviewed about their confidence in delegating the supply of NPMs to pharmacy staff all pronounced their confidence in these staff for the role with the knowledge that if unsure the staff would approach the pharmacists for clarification and advice. ${ }^{48}$ Evidence of this in practice can been seen where medicine counter assistants observed showed that referrals to pharmacists from pharmacy staff was largely for gastrointestinal symptoms when compared with respiratory or ear, nose and throat symptoms, the pharmacy staff felt more knowledgeable in dealing with the latter two and they felt these conditions were less serious than gastrointestinal; symptoms. ${ }^{16,17}$

Studies that compared outcomes of various aspects of consultations for NPMs by considering which member of the pharmacy team was involved in the consultation revealed that pharmacists tended to perform better than pharmacy staff. $16,19,34,37,38,64$ Although 
observational data showed that the involvement of pharmacists in consultations for NPMs were generally opportunistic and unplanned in nature, pharmacists provided advice in almost twice the number of consultations when compared with pharmacy staff. 16 Pharmacists performed significantly better in questioning people and eliciting information from them, ${ }^{63}$ and pharmacists conducted more guideline-compliant consultations. ${ }^{63-65}$ Another observation study showed that significantly more consultations for medicines sales that involved pharmacists were accompanied by counseling when compared with consultations carried out by pharmacy staff. ${ }^{19}$ Pharmacists also achieved higher consultations scores when involved in responding to symptoms when compared with pharmacy staff. ${ }^{37}$ Pharmacists were found to have a higher chance of identifying and commenting on interactions. 34

Where consultations involved both the pharmacist and pharmacy staff, observation studies revealed that these consultations were performed much better than with any pharmacy team member alone. ${ }^{16,64}$ In one study, $97 \%$ of consultations that involved both pharmacist and pharmacy staff saw customers receiving advice. ${ }^{16}$ Teaming of pharmacists and pharmacy staff resulted in a higher proportion of appropriate sales or compliant consultations compared to either group of staff carrying out these consultations on their own. ${ }^{63-65}$

\section{Responding to symptoms versus direct product requests}

Although studies reviewed suggested that consultations for product requests outnumbered those that involved symptom presentation 65,84 it was in fact consultations that involved symptom presentation that were conducted to a higher standard when compared with those where someone asked directly for a product. $37,38,65,84,91$ Mystery shopper with feedback intervention studies showed that although overall consultation scores improved following the intervention, responding to symptom consultations scored higher than direct product requests. 37,38 However, in terms of improvement from baseline, the consultations for direct products requests improved more than consultations for responding to symptoms. ${ }^{38}$ Compliance with guideline recommendation was more evident when dealing with scenarios involving symptom presentation compared to product requests. ${ }^{65}$ Pharmacists reported that they felt they had more control and flexibility when coming to making decisions of product to supply when people presented symptoms rather than asking for a specific product. 84

A mystery shopper with feedback intervention study ${ }^{39}$ indicated that communication within consultations involved with responding to symptoms was significantly much better than in direct product request consultations in terms of length of sentence, use of open-ended questions, overall impression of counseling service.

\section{Brand in mind or no brand in mind}

A vast majority of people visited a pharmacy to purchase a medicine when they already had 
a particular brand in mind. ${ }^{41,42}$ A majority of these people proceeded to buy the medicine they had in mind. Approximately one-fifth of these people bought the medicine without a consultation. 42 The majority of these who had brand in mind asked the pharmacy staff for the medicine and approximately a tenth self-selected the product themselves without first consulting pharmacy staff. ${ }^{41}$ On the contrary, when people made a visit to the pharmacy without a particular brand in mind, most of the medicine sales that followed resulted from the person making some form of request, although a small percentage of these people made an unguided selection from the product shelves. 41,42

\section{Time available or allocated to consultations for NPMs}

Interviews conducted with pharmacists following a health promotion campaign carried out in the pharmacy revealed that pharmacists felt that the pharmacy staff team did not have enough time to speak with people. ${ }^{35}$ In sales of medicines governed by standards for the provision of Pharmacist Only and Pharmacy Medicines in community pharmacy (S2/S3 standards), sales for S3s (where pharmacists had to be involved) pharmacists reported not having enough time to attend to every request for an S3 and at times limited their involvement to verifying standardized tick box forms completed by support staff to confirm the therapeutic appropriateness of treatment being proposed to be supplied to the customer, therefore there input in supply of $\$ 3$ was at times very limited. 48

An assessment of how pharmacists used their time in community pharmacy showed less time was devoted to their involvement with consultations for NPMs compared to the time devoted to completing mechanical tasks, not requiring the expertise of the pharmacist, such as product assembly and labeling. 15,75 The activity that pharmacists gave their least amount of time to was staff training. ${ }^{15,75}$

Studies that involved work sampling showed that even when the pharmacy was facing a busy period, the time spent providing advice on NPMs remained steady. ${ }^{26}$ However, the volume of prescriptions dispensed in a pharmacy impacted how much time pharmacists engaged in counseling for the supply of NPMs or responding to symptoms. Significantly less time was spent on these activities in pharmacies with higher volumes of prescriptions dispensed. ${ }^{15,75}$ An intervention study using mystery shoppers resulted in people receiving significantly longer consultations with counseling times increasing to $5.1 \mathrm{~min}$ from the original 3.6 min before the mystery shopper visit. ${ }^{76}$ Interviews conducted following a service evaluation reported that pharmacy staff believed that people would benefit if there was more interaction with the pharmacy staff and if more time is spent on the consultation. 57

\section{Attributes of community pharmacy where NPMs are supplied}

Community pharmacists were viewed by some people as medicine experts 47 and they felt that pharmacy staff had the expertise required to deal with their medical complaints. 85 Involving pharmacy staff in an intervention that involved identifying product misuse led to 
raising awareness amongst them and made them realize their vital role they had in supporting patients to use medicine safely. 68

Some customers felt that community pharmacy served the same function as the GP. ${ }^{11}$ People reported that the approachability of pharmacy staff and their ability and willingness to spend more time dealing with them was a positive of visiting the community pharmacy. 47

People used the community pharmacy instead of visiting the GP as a way to reduce GP workload or prevent unnecessary workload on GPs. $21,46,47,57,88$ At times when people were not satisfied with the outcome of their GP visit the community pharmacy was seen as the place to go as an alternative to the GP.10,11,47 One pharmacy design was intentional in displaying $\mathrm{P}$ medicines as dummy packs where people could self-select them as a way of engaging people more in the decision-making of purchase of NPMs and was reported to have improved access and engagement. 61

Using community pharmacy was seen as a way to improve access to care, it was said to improve peoples' choices (e.g. compared to what products the GP could offer), 27,57 and it was seen as a way of channeling people to the right care setting. 47

The community pharmacy setting was used as it was reported to be convenient $21,27,47,57,67,85,88$ and time-saving. 27,67 People reported using the community pharmacy out of habit. 85 Community pharmacies were used due to ease of access, which for some this specifically meant not needing to make an appointment $21,27,47$ or not having to wait. ${ }^{27}$ An additional advantage reported was the benefit of being able to have consultations and medical issues attended to 'by proxy.'21,47,72

In some cases community pharmacy was seen as the affordable option for people. $21,27,57$ Buying NPMs worked out as a cheaper alternative in some instances. 27

Overall, pharmacists and pharmacy staff were commended for their good communication skills ${ }^{14,39}$ and interpersonal skills. ${ }^{22}$ At times standard of communication was concluded to be excellent by those who mystery shopped pharmacies. ${ }^{12}$ Some mystery shoppers described the pharmacist's style of communication as empathetic. ${ }^{86}$ Pharmacists reported adapting their communication style and interacted with people in response to nonverbal signs (reading expressions, appearance of the eyes and specific motions) given by people during consultations. ${ }^{49}$ Pharmacists realized their responsibility for lifelong learning involved with pharmacy even with regards to an area such as communication, they identified that they were responsible to maintain their training and keep up to date. 61 Being able to speak and share the same language as people form a minority ethnic background was viewed as an important role of pharmacy staff from the same minority ethnic background. Having a shared language facilitated in communicating details of a folic acid health promotion in the pharmacy with people with whom they shared the same 
ethnicity and language. 35

On the contrary to the positive aspects of community pharmacy highlighted above, negative features that were reported, including some people who felt that it was embarrassing to have to discuss their ailments at the pharmacy, 41,67 and a lack of privacy. ${ }^{41,60,67}$ The overall layout of the community pharmacy setting was also seen as a barrier to communication. 60 People reported that communicating with pharmacy staff was not always that easy, as pharmacists at times appeared to be busy, 60,85 and perhaps more busy than they really were as a strategy to avoid communicating with people 60 and hence were seen as unapproachable.

Some people did not believe NPMs to be potent enough and preferred to obtain more potent ones from their GP. ${ }^{27}$ In fact, an interview study of the use of evidence based medicine by pharmacists in the area of NPMs revealed that discussing the lack of evidence of the effectiveness of NPMs was not easy for pharmacists and reflected on the ethics of selling NPMs were evidence of effectiveness has not been confirmed. ${ }^{83}$ They explained that reasons that made it uncomfortable to discuss the lack of evidence of the effectiveness included dealing with product popularity and not wanting to destroy peoples' confidence in a particular product. Furthermore, an additional challenge put forth was the possible conflict of interest in using evidence based practice as the community pharmacy was in fact a retail business. 83

There appeared to be a lack of awareness and confusion about the role of the community pharmacy, and it was the view of some people that it was not in the pharmacist's area of expertise to be involving themselves with diagnosis illnesses 47 or medicines management ${ }^{60}$; rather, their role was mainly that of filling prescriptions ${ }^{60}$ and pharmacist were not to interfere where medicines are recommended by the GP60,85; the GPs were the ones who possessed the knowledge. 85

\section{Discussion}

\section{Discussion of key findings}

Numbers of studies to review increase year on year and the trend over the last 5 years indicates that the number of studies relevant to the supply of NPMs in community pharmacies continues to rise. The majority of the studies were conducted in the UK and Ireland and the second largest group of studies covered the Australia and New Zealand which have a similar community pharmacy context to the UK. Scoping studies further afield from other European countries and North America helped shed some insight into the realities to be considered if NPMs are made available for self-selection. Studies included in the review were often small and of low quality with small sample sizes. If interventions and practice changes are derived from methodologically weak interventions they may be implemented or rejected in error. It may seem surprising that more large studies about pharmacist supply of NPMs have not been conducted. However, pharmacy chains have not 
had a history of conducting research, and research training for community pharmacists and research networks did not exist until fairly recently. The recent growth of research networks and the increasing amount of research being conducted by pharmacy chains in the UK and beyond is encouraging and may led to an increase in larger higher quality national and international studies which would have been difficult to conduct before.

\section{Is the pharmacist important in the supply of NPMs?}

The primary aim of this systematic review was to identify and summarize the available evidence of the role pharmacists play in maintaining and guaranteeing patient safety and improving the quality of consultations for supply of $\mathrm{P}$ medicines, and to establish whether or not the intervention of a pharmacist is important in the sale of $\mathrm{P}$ medicines in community pharmacies. Although some studies did exclusively include pharmacists $5,12,14,18,22,26,28-$ $30,40,43,48,49,53,57-59,61,69,75-77,79,81,83,84,86$ as participants in studies undertaken and in some settings pharmacists did not have support staff, it was evident from most of the studies reviewed that non-pharmacist staff featured largely in consultations for NPMs and play a significant role in the supply of NPMs. ${ }^{16,17,31,63}$ It is interesting that pharmacists tended to perform better than pharmacy staff $16,19,34,37,38,64$ and provide advice in almost twice as many consultations ${ }^{16}$ suggesting that further training and better use of each of the pharmacy team's skills is needed. Perhaps pharmacists need to spend more time responding to customers' symptoms and supplying NPMs. These findings clearly indicate the value of having pharmacists involved in supply of NPMs.

Pharmacists and pharmacy staff were commended for good communication skills $12,14,39$ and interpersonal skills. ${ }^{22,86}$ However, there was evidence for need to improve all aspects of consultations and a need to be consistent throughout consultations, as all aspects play a unique role in enabling appropriate use of NPMs. Information-gathering by pharmacists and pharmacy staff through questioning people is not always carried out comprehensively, 16,65 with factors such as the pharmacy environment, $41,60,67$ lack of

elsevier_RSAP_717

time, lack of understanding of protocols and poor communication skills being mentioned as contributors. There is scope to improve the pharmacy staff's questioning behavior through the use of interventions such as mystery shopper with feedback. ${ }^{87}$ Such methods could be more widely used to improve performance. The development of communication and consultation skills should be prioritized so that pharmacists can understand and engage with the patient's own discourse. So for example, a patient may have a different view of risk from the pharmacist so the pharmacist needs to help the patient understand the risk in such a way the patient understands how to take the medicines and is not put off doing so. Also training programs as presented in the papers, ${ }^{87}$ should not be seen as one off events but an on going quality improvement process.

Having to extract information from unwilling people or people who are not privy to the information such as visits to the pharmacy made by proxy $21,47,72$ has also been highlighted 
as a barrier to information gathering. Notwithstanding this, the fact that effective information gathering is a prerequisite to an effective consultation process outcome including; provision of NPM, refusal to provide NPM or referral, means that ways to overcome these barriers are necessitated. Ways to improve the pharmacy environment for example, busyness, 60,85 staff levels, competing demands, enhancing pharmacy staff skills and knowledge, and means of engaging people by explaining the importance of gathering correct information and explaining why information is being asked, may also prove to be helpful. ${ }^{60,85}$ If more NPMs move to self-selection, more onus is likely to be placed on people to initiate the first contact with pharmacy staff. If people select an inappropriate treatment, for some the result may be negative health outcomes and could lead to prolongation of ailment, deterioration of condition and suffering unwanted medicine effects.

Although staff were able to identify and deal with drug related problems $51,69,72,79$; in some instances drug issues were missed by pharmacy staff. ${ }^{34}$ One study showed that drugrelated problems were $60 \%$ higher in the pharmacies selling NPMs over the counter than if just sold via self selection. ${ }^{69}$ This indicates the value of having pharmacist and pharmacy staff involvement in supply rather than just self selection.

Pharmacists have a multifaceted role in supply of $\mathrm{P}$ medicines. People have varying expectations along the spectrum of expecting questions, advice and information from pharmacists and their staff to expecting to be able to select a product, purchase it and leave without being asked or asking any questions. As Banks 92 and Stevenson 61 have discussed, all of this takes place in an environment that is rooted in both health and commerce. There is an interesting balance to strike between treating people as patients or customers who know what they wish to purchase and enabling them to experience health care and be empowered for self care. There is a lack of health literacy around pharmacy medicines, and if medicines were only available for self-selection or on prescription, people would need to be much more educated than at present about the need to seek advice, particularly if they have existing long term conditions for which they are taking other medicines. Medicines are not like any other commercial product. The need to refer people in an appropriate and timely manner to a more suitable health care such as the GP strengthens the case for needing meaningful interactions to happen between customers and pharmacy staff for treating minor ailments with NPMs.

Some products recommended in community pharmacy were deemed to be inappropriate. $51,69,72,79$ This review identified community pharmacy as both a main source of products that are misused but also a setting that provided a unique position to identify misuse. ${ }^{78,87}$ The identification of misuse depends on knowing/seeing/being familiar with customers, which in turn was made possible where a pharmacy had a stable workforce especially of frontline staff. This makes adequate and regular staffing of community pharmacies an item of importance for patient safety. Because noticing the repeat buying of the same product can help identify misuse questions should be asked of pharmacy settings, such as some supermarkets, which currently display medicines at distances away from the actual pharmacy. Inconsistencies in process outcome for customers when they visit different pharmacies may be particularly unhelpful where medicines are 
suspected to be being abused. Therefore, pharmacy staff should be consistent in the criteria they use to refuse a sale for an OTC that is suspected as being misused/abused, patients should be receiving the same message irrespective of which pharmacy they visit and there should not be a tier of 'easy' and hard pharmacies or staff for which inappropriate supplies of medicines are made. Adherence to protocols and guidelines may be a useful aid to achieve this consistency. If interaction with customers is removed by self-selection it can make it harder to identify misuse and provide support and guidance to customers. In the interest of patient safety extra restrictions (even if by proximity) should be considered for medicines which are likely to be misused if a move to self-selection is recommended.

\section{Provision of information}

While pharmacists were identified as the predominant source of information for NPMs. 62 There was a large number of instances where no advice and/or information provision was recorded. ${ }^{16}$ Provision of information and advice is a crucial element of consultations for NPMs which in light of protocols of sales of NPMs would be deemed unsatisfactory. On the other hand, the review showed that when information and advice was imparted customers did not retain much of it anyway which provides a challenging paradox. Where written information was given, one study reported that two-thirds of people did not read it. ${ }^{9}$ This emphasizes the importance for provision of verbal information at the time of supply. Of note was the fact that people did appear to recall advice that was repeated and that constituted a procedural piece of advice/instruction. ${ }^{18}$ Intervention studies have shown that pharmacy staff advice ${ }^{71}$ and goal setting 55,89 lead to greater symptom relief.

In reality this may mean that pharmacy staff and protocol developers need to acknowledge that overloading people with information for the sake of adhering to a protocol is not going to be useful, but consideration may be given to develop protocols and consultation styles that include intentional repetition of key information to communicate. Ascertaining what existing knowledge someone holds about an NPM may help tailor the conversation to focus on 'new' areas. As it is not possible to equip people with all the necessary product information and advice in one consultation, verbal information supplemented with either written information or highlighting reputable sources of information found on the internet may prove useful. Making opportunities to speak to people who make repeated visits to the pharmacy provides an avenue to build their product knowledge, whereas this review implied that for these people's knowledge is sometimes assumed due to frequency of purchasing a product, which in fact should perhaps alert pharmacy staff of further intervention being warranted. In addition to this, people may need to be alerted to changes in product advice, for example the change of dose of paracetamol in children, a product many people declared their confidence in using due their long experience with this product. Similar to information-gathering, a move to self-selection may result in reduced opportunities to interact with people and will place the individual in the driving seat to seek information and advice, which is something that should be encouraged.

\section{Referral}

The need to refer people in an appropriate and timely manner to a more suitable health 
care provider such as the GP, as identified in the review, further strengthens the case for needing meaningful interactions to happen between people and pharmacy staff for treating minor ailments with NPMs. As this review shows, people do not necessarily correctly recall advice to see for instance the GP 18 but also even when they have grasped this piece of advice they do not necessarily act on it. ${ }^{18,49,70}$ Once again the issue of pharmacy staff needing to clearly communicate with people (with repetition) the reason for referral must be stressed. It may even be prudent for urgent referrals instigated by pharmacy staff to be followed up by the pharmacist.

\section{Symptom response versus direct product request}

Consultations for symptom response are dealt with more effectively than direct product requests. $37,38,65,84,91$ This is partly due to the fact that people making a direct product request know what they want and staff appear to feel uncomfortable to exercise unsolicited questions and to provide unsolicited advice. This strongly supports the findings in this review that indicate that the success of consultations and facilitation of appropriate sales is dependent and is better effected when people are engaged in all aspects of the consultation. This includes people knowing and being willing to engage the pharmacy team when they have the ability to self-select a product. More awareness than this can help staff recognize the challenge but perhaps training for dealing with NPMs requires two models of consultation styles or protocols rather than the usual focus on attempting to cover a set of questions and deliver advice and information, but rather have one model for responding to symptoms and one for direct product request, this focus has already been adopted by some pharmacy chains.

Pharmacists and non-pharmacist staff make a number of key contributions that would be unlikely to happen if medicines moved to self-selection, and this may lead to delayed diagnoses and delayed referrals. Delays ${ }^{67}$ in giving advice and information which has been shown can have a positive effect on consumer outcome as well as identifying and resolving drug related problems included drug misuse/abuse. If $\mathrm{P}$ medicines are to be made available for self-selection, processes to ensure that patients receive a pharmacist's intervention when appropriate will need to be considered.

It is important that Pharmacist and non-pharmacist staff improve and maintain their skills in responding to people's symptoms and product selection for NPMs as product availability and advice changes. ${ }^{61}$ Regular training is needed on newly deregulated products and changes in product advice. Very little time appeared to be dedicated to training nonpharmacist staff. 15,75

\section{Interventions to improve consultations for NPMs}

Opportunities to capitalize on interventions that have shown promise in improving consultations for NPMs should be sought for example, targeted training and quality improvement initiatives such as those employed in Australia 53 and piloted in Scotland 66 are needed to further develop pharmacists' skills to ensure the appropriate supply of $P$ medicines. Many of the studies did not distinguish between pharmacy staff and 
pharmacists; however, in the UK pharmacists do have the ultimate responsibility for sale of $\mathrm{P}$ medicines, and it is important that they can intervene in sales and be available to advice and answer patient questions.

\section{Strengths and limitations}

Reviewing studies in the English and the Dutch language may have narrowed the final number of studies identified for the review.

Restricting studies to those conducted in the UK and similar countries although useful for being able to summarize data that is close to the context of interest i.e. UK may have narrowed the opportunity to explore how NPMs are handled in different contexts, which would benefit the pharmacy team, as UK is a multicultural society, and customers visiting pharmacies may have in fact originated from settings outside the UK. Understanding their context may have helped predict their response or behavior if proposal to make medicines accessible by self-selection is realized.

The limitations of available studies investigating the role of the pharmacist specifically impacted fulfilling the aim of the study, and identifying role of pharmacists separate from other pharmacy team members was not always achievable. The decision to present results for community pharmacy staff as a team rather than focusing solely on the pharmacists was necessitated due to the fact that pharmacy staff were identified frequently as making significant contributions to consultations for NPM. Additionally, a number of studies did not separate out the contribution of the pharmacist from other staff, making it impossible to clearly identify what unique role the pharmacist may have played.

The quality of a consultation for an NPM is a multifaceted concept and as evident from this review different aspects were assessed to gauge the 'quality' of a consultation. Having one measure to assess quality for consultations for NPMs would make assessment of quality more manageable however this is an unrealistic expectation as community pharmacies as a setting are a multifaceted health setting environment.

Reviewing studies with different study designs added to the complexity of summarizing findings, solely focusing on one study design type such as interventions may have been able to give a clearer picture of whether quality can be improved. Nonetheless, the weakness of this study is also a strength in that by using a variety of study designs not only has what actually happens in consultations has been able to be captured (e.g. through observation studies) but also what pharmacy staff and customers perceive happen, perceptions may influence the behavior of how both pharmacy staff and customers interact in a consultation for an NPM. New studies need to be commissioned to generate a more homogenous body of evidence.

\section{Conclusion}

The primary aim of this systematic review was to identify and summarize the available evidence of the role pharmacists play in maintaining and guaranteeing patient safety, minimizing abuse of NPMs and improving the quality of consultations for supply of $P$ medicines, and to establish whether or not the intervention of a pharmacist is important in 
the sale of $\mathrm{P}$ medicines in community pharmacies. If pharmacy staff lack the competence to carry out effective NPM consultations, the rationale for keeping NPMs behind the counter is somewhat unfounded. However, the positive contribution made by pharmacy staff in the supply (or, refusal of supply) of NPMs cannot be entirely ignored, so continued efforts to enhance this service even in its current model should to be supported. Whether selfselection becomes a reality in UK pharmacies, there still appears to be a need to up-skill pharmacy staff so that consultations for NPMs are of better quality.

The important role that customers play in enabling successful consultations for NPMs should not be underestimated. Seeking opportunities to communicate with customers that pharmacy staff rely on there being a minimal level of information exchange for appropriate and safe supply of NPMs to happen, may result in a more positive view amongst people to unsolicited questioning and information/advice giving from staff. An important consideration for pharmacy owners and organizations is therefore the provision of a conducive environment for optimal information exchange to occur, where staff levels, time and space are adequate.

This review was based on 83 papers, with many different study designs and methods of measuring outcomes, thus making comparisons difficult. The pharmacist and their staff have so many different roles and use a number of different skills in the process of supply of NPMs, and not everything can be rigidly defined. To overcome these problems, larger studies should be commissioned to generate a more homogenous body of evidence.

\section{Acknowledgements}

This work was funded by the Royal Pharmaceutical Society and School of Pharmacy, University of Nottingham

References

1 Anon. United Kingdom. Medicines Act 1968. England: Harry Pitchforth; 1968.

2 Society RP, Medicines, Ethics and Practice, 2015, Royal Pharmaceutical Society; London.

3 Legal Status and Reclassification. at:

http://www.mhra.gov.uk/Howweregulate/Medicines/Licensingofmedicines/Legalstatusandreclassification/; Accessed 07.12.15. 4

4 Types of Medicines, 2015, at: http://www.rpharms.com/ipharmacist/types-of-medicine.asp, Accessed 07.12.15. 5

5 Open Display of Pharmacy Medicines: What the GPhC Says, 2013, at: http://www.pharmacyregulation.org/open-display-pharmacy-medicines-what-gphc-says, Accessed 07.12.15. 6

6 A. Liberati, D.G. Altman, J. Tetzlaff, et al., The PRISMA statement for reporting systematic reviews and metaanalyses of studies that evaluate health care interventions: explanation and elaboration, PLoS Med 6, 2009, e1000100. 7

7 K. Khan, R. Kunz, J. Kleijnen and G. Antes, In: 2nd ed, Systematic Reviews to Support Evidence Based Medicine 2011, Hodder Arnold; London. 
8 Methods for the Development of NICE Public Health Guidance, 2012, National Institute for Health and Care Excellence, at: http://www.nice.org.uk/article/pmg4/resources/non-guidance-methods-for-the-developmentof-nice-public- health-guidance-third-edition-pdf, Accessed 07.12.15.

9 A.T.G. Blom and J.A.L. Rens, Information about over-the-counter medication: the role of the pharmacy, Patient Educ Couns 14, 1989, 181-189.

$10 \mathrm{~S}$. Cunningham-Burley and U. Maclean, The role of the chemist in primary health care for children with minor complaints, Soc Sci Med 24, 1987, 371-377.

11 S. Cunningham-Burley and U. Maclean, Pharmacists and primary care: some research findings and recommendations, Fam Pract 5, 1988, 122-125.

12 H.V. Feehan, Evaluating pharmacist competency: self care products, Contemp Pharm Pract 4, 1981, 115120.

13 P.J. Kiernan and J.B. Issacs, Use of drugs by the elderly, J R Soc Med 74, 1981, 196-200.

14 C.W. Barnett, D. Nykamp and W.A. Hopkins, Evaluation of pharmacists' OTC drug consultations, J Pharm Mark Manage 6, 1992, 33-49.

15 H.M. Bell, J.C. McElnay and C.M. Hughes, A self-reported work sampling study in community pharmacy practice, Pharm World Sci 21, 1999, 210-216.

16 P. Bissell, P.R. Ward and P.R. Noyce, Variation within community pharmacy. 1. Responding to requests for over-the-counter medicines, J Soc Adm Pharm 14, 1997, 1-15.

17 P. Bissell, P.R. Ward and P.R. Noyce, Variation within community pharmacy. 2: responding to the presentation of symptoms, J Soc Adm Pharm 14, 1997, 105-115.

18 S.W. Evans, D.N. John, M.J. Bloor and D.K. Luscombe, Use of non-prescription advice offered to the public by community pharmacists, Int J Pharm Pract 5, 1997, 16-25.

19 C.M. Fisher, O.I. Corrigan and M.C. Henman, A study of community pharmacy practice. 3. Non-prescribed medicines sales and counselling, J Soc Adm Pharm 8, 1991, 69-75.

20 K. Hassell, P. Noyce, A. Rogers, J. Harris and J. Wilkinson, Advice provided in British community pharmacies: what people want and what they get, J Health Serv Res Policy 3, 1998, 219-225.

21 K. Hassell, P.R. Noyce, A. Rogers, J. Harris and J. Wilkinson, A pathway to the GP: the pharmaceutical 'consultation' as a first port of call in primary health care, Fam Pract 14, 1997, 498-502.

22 G.D. Lamsam and M.A. Kropff, Community pharmacists' assessments and recommendations for treatment in four case scenarios, Ann Pharmacother 32, 1998, 409-416.

23 J. Lilja and S. Larsson, Mental mirrors of pharmacists: how do pharmacists perceive their over-the-counter customers?, Int J Pharm Pract 2, 1993, 136-141.

24 B. Marklund, G. Karlsson and C. Bengtsson, The advisory service of the pharmacies as an activity of its own and as part of a collaboration with the primary health care services, J Soc Adm Pharm 7, 1990, 111-116.

25 C.J. Morris, J.A. Cantrill and M.C. Weiss, 'One simple question should be enough': consumers' perceptions of pharmacy protocols, Int J Pharm Pract 5, 1997, 64-71.

26 I. Savage, Time for prescription and OTC advice in independent community practice, Pharm J 258, 1997, 
873-877.

27 E.I. Schafheutle, J.A. Cantrill, M. Nicolson and P.R. Noyce, Insights into the choice between self-medication and a doctor's prescription: a study of hay fever sufferers, Int J Pharm Pract 4, 1996, 156-161.

28 O.E. Sierralta and D.M. Scott, Pharmacists as nonprescription drug advisors, Am Pharm NS35, 1995, 36-38.

29 F.J. Smith, A study of the advisory and health promotion activity of community pharmacists, Health Educ J 51, 1992, 68-71.

30 F.J. Smith and M.R. Salkind, Presentation of clinical symptoms to community pharmacists in London, J Soc Adm Pharm 7, 1990, 221-224.

31 J.R. Sowter and D.K. Raynor, Management of oral health problems in community pharmacies, Pharm J 259, 1997, 308-310.

32 J. Taylor and L. Suveges, Selection of cough, cold, and allergy products. Rate of consumer-pharmacist interaction, J Soc Adm Pharm 9, 1992, 59-65.

33 J. Taylor and L. Suveges, Frequency of consumer-pharmacist interaction during the selection of nonprescription medications, Can Pharm J 127, 1994, 27-30.

34 D. Alte, W. Weitschies and C.A. Ritter, Evaluation of consultation in community pharmacies with mystery shoppers, Ann Pharmacother 41, 2007, 1023-1030.

35 C. Anderson and R. Rajyaguru, The role of community pharmacists and medicines counter assistants in health promotion: reflections from a folic acid campaign, Int J Pharm Pract 10, 2002, 17-22.

36 .Å.C. Andersson, H. Brodin and J.L.G. Nilsson, Pharmacist interventions in relation to patient drug-related problems, J Soc Adm Pharm 20, 2003, 82-91.

37 S.C. Benrimoj, A. Gilbert, N. Quintrell and A.C. Neto, Non-prescription medicines: a process for standards development and testing in community pharmacy, Pharm World Sci 29, 2007, 386-394.

38 S.I. Benrimoj, J.B. Werner, C. Raffaele, A.S. Roberts and F.A. Costa, Monitoring quality standards in the provision of non-prescription medicines from Australian Community Pharmacies: results of a national programme, Qual Saf Health Care 16, 2007, 354-358.

39 K. Berger, C. Eickhoff and M. Schulz, Counselling quality in community pharmacies: implementation of the pseudo customer methodology in Germany, J Clin Pharm Ther 30, 2005, 45-57.

40 H.C. Diener, A. Dowson, S. Whicker and T. Bacon, Development and validation of a pharmacy migraine questionnaire to assess suitability for treatment with a triptan, J Headache Pain 9, 2008, 359-365.

41 L. Emmerton, Behavioural aspects surrounding medicine purchases from pharmacies in Australia, Pharm Pract 6, 2008, 158-164.

42 L.M. Emmerton, Exploration of two risk scenarios in non-prescription medicine purchases, Int J Pharm Pract 14, 2006, 219-225.

43 G.F. Fleming, J.C. McElnay and C.M. Hughes, Development of a community pharmacy-based model to identify and treat OTC drug abuse/misuse: a pilot study, Pharm World Sci 26, 2004, 282-288.

44 A.G. Granas, A. Haugli and A.M. Horn, Smoking cessation advice provided in 53 Norwegian pharmacies, Int J Pharm Pract 12, 2004, 179-184. 
45 S. Gray, S. Woolfrey, R. Copeland, D. Gill and G. Dennett, Evaluating the potential impact of community pharmacy interventions on patient care in Northumberland, Qual Prim Care 12, 2004, 47-51.

K. Hassell, Diverted traffic, Health Serv J 6, 2000, 22-23.

47 K. Hassell, A. Rogers and P. Noyce, Community pharmacy as a primary health and self-care resource: a framework for understanding pharmacy utilization, Health Soc Care Community 8, 2000, 40-49.

48 H.L. Hattingh, M.A. King and N.A. Smith, An evaluation of the integration of standards and guidelines in community pharmacy practices, Pharm World Sci 31, 2009, 542-549.

49 N.M. Katajavuori, S.P. Valtonen, K.M. Pietilä, A.O. Pekkonen, S.A. Lindblom-Ylänne and M.S. Airaksinen, Myths behind patient counselling: a patient counselling study of non-prescription medicines in Finland, J Soc Adm Pharm 19, 2002,

129-136.

50 F.S. Kelly, K.A. Williams and S.I. Benrimoj, Does advice from pharmacy staff vary according to the nonprescription medicine requested?, Ann Pharmacother 43, 2009, 1877-1886.

51 M.C. Laliberte, M. Normandeau, A. Lord, et al., Use of over-the-counter medications and natural products in patients with moderate and severe chronic renal insufficiency, Am J Kidney Dis 49, 2007, 245-256.

52 G. March, M. Annells and T. Koch, Older people, constipation and laxative use: management implications, J Soc Adm Pharm 19, 2002, 190-199.

53 A.C.D. Neto, S.I. Benrimoj, D.J. Kavanagh and R.A. Boakes, Novel educational training program for community pharmacists, Am J Pharm Educ 64, 2000, 302-307.

54 P.T. Norris, Purchasing restricted medicines in New Zealand pharmacies: results from a "mystery shopper" study, Pharm World Sci 24, 2002, 149-153.

55 .J. O'Connor, C. Seeto, B. Saini, et al., Healthcare professional versus patient goal setting in intermittent allergic rhinitis, Patient Educ Couns 70, 2008, 111-117.

56 R. Peters, Jr., G.S. Yacoubian, Jr., W. Rhodes, et al., Beliefs and social norms about codeine and promethazine hydrochloride cough syrup (CPHCS) use and addiction among multi-ethnic college students, $J$ Psychoactive Drugs 39, 2007, 277-282.

57 S. Pumtong, H.F. Boardman and C.W. Anderson, Pharmacists' perspectives on the pharmacy first minor ailments scheme, Int J Pharm Pract 16, 2008, 73-80.

58 I.I. Puumalainen, S.H. Peura, H.M. Kansanaho, C.S.I. Benrimoj and M.S.A. Airaksinen, Progress in patient counselling practices in Finnish community pharmacies, Int J Pharm Pract 13, 2005, 149-156.

59 P.M. Rutter, E. Horsley and D.T. Brown, Evaluation of community pharmacists' recommendations to standardized patient scenarios, Ann Pharmacother 38, 2004, 1080-1085.

60 M.L. Skomo, S.P. Desselle and N. Shah, Migraineurs' perceptions of and interactions with pharmacists: a qualitative study, Int J Pharm Pract 16, 2008, 357-363.

61 F.A. Stevenson, M. Leontowitsch and C. Duggan, Over-the-counter medicines: professional expertise and consumer discourses, Sociol Health IIIn 30, 2008, 913-928.

62 J. Tio, A. LaCaze and W.N. Cottrell, Ascertaining consumer perspectives of medication information sources 
using a modified repertory grid technique, Pharm World Sci 29, 2007, 73-80.

63 M.C. Watson, C.M. Bond, J. Grimshaw and M. Johnston, Factors predicting the guideline compliant supply (or non-supply) of non-prescription medicines in the community pharmacy setting, Qual Saf Health Care 15, 2006, 53-57.

64 M.C. Watson, C.M. Bond, J.M. Grimshaw, J. Mollison, A. Ludbrook and A.E. Walker, Educational strategies to promote evidence-based community pharmacy practice: a cluster randomized controlled trial (RCT), Fam Pract 19, 2002, 529-536.

65 M.C. Watson, C.M. Bond, M. Johnston and K. Mearns, Using human error theory to explore the supply of nonprescription medicines from community pharmacies, Qual Saf Health Care 15, 2006, 244-250.

66 M.C. Watson, J.A. Cleland and C.M. Bond, Simulated patient visits with immediate feedback to improve the supply of over-the-counter medicines: a feasibility study, Fam Pract 26, 2009, 532-542.

67 M.C. Watson, A.E. Walker and C.M. Bond, Community pharmacists' views and beliefs about the treatment of symptoms suggestive of vaginal thrush in community pharmacies, Pharm World Sci 22, 2000, 130-135.

68 M. Wazaify, C.M. Hughes and J.C. McElnay, The implementation of a harm minimisation model for the identification and treatment of over-the-counter drug misuse and abuse in community pharmacies in Northern Ireland, Patient Educ Couns 64, 2006, 136-141.

69 L.O.T. Westerlund, B.R.G. Marklund, W.H.A. Handl, M.E. Thunberg and P. Allebeck, Nonprescription drugrelated problems and pharmacy interventions, Ann Pharmacother 35, 2001, 1343-1349.

70 T. Westerlund, P. Allebeck, B. Marklund, I.L. Andersson, J.O. Branstad and M. Sjoblom, Evaluation of a model for counseling patients with dyspepsia in Swedish community pharmacies, Am J Health Syst Pharm 60, 2003, 1336-1341.

71 T. Westerlund, I.L. Andersson and B. Marklund, The quality of self-care counselling by pharmacy practitioners, supported by IT-based clinical guidelines, Pharm World Sci 29, 2007, 67-72.

72 I.C.K. Wong, S.C. Siew and H. Edmondson, Children's over-the-counter medicines pharmacoepidemiological (COPE) study, Int J Pharm Pract 15, 2007, 17-22.

73.Z.U.D. Babar, K. Pengelly, S.L. Scahill, S. Garg and J. Shaw, Migrant health in New Zealand: exploring issues concerning medicines access and use, J Pharm Health Serv Res 4, 2013, 41-49.

74 C. Bardage, T. Westerlund, S. Barzi and C. Bernsten, Non-prescription medicines for pain and fever-A comparison of recommendations and counseling from staff in pharmacy and general sales stores, Health Policy 110, 2013, 76-83.

75 L. McCann, C.M. Hughes and C.G. Adair, A self-reported work-sampling study in community pharmacy practice: a 2009 update, Pharm World Sci 32, 2010, 536-543.

76 T. Bertsche, M. Nachbar, J. Fiederling, et al., Assessment of a computerised decision support system for allergic rhino-conjunctivitis counselling in German pharmacy, Int J Clin Pharmacol 34, 2012, 17-22.

77 H.F. Boardman, N.J. Gray and B.S. Symonds, Interactions between parents/carers of pre-school children and pharmacy staff when buying non-prescription medicines, Int J Clin Pharmacol 33, 2011, 832-841.

78 R. Cooper, Surveillance and uncertainty: community pharmacy responses to over the counter medicine abuse, Health Soc Care Community 21, 2013, 254-262. 
79 C. Eickhoff, A. Hammerlein, N. Griese and M. Schulz, Nature and frequency of drug-related problems in self-medication (over-the-counter drugs) in daily community pharmacy practice in Germany,

Pharmacoepidemiol Drug Saf 21, 2012, 254-260.

80 J. Gordon, J.P. Calabretto and M.J. Sorich, Unanswered health-related questions in community pharmacy: frequency, nature and consequences, J Pharm Pract Res 41, 2011, 271-274.

81 N.J. Gray, H.F. Boardman and B.S. Symonds, Information sources used by parents buying non-prescription medicines in pharmacies for preschool children, Int J Clin Pharmacol 33, 2011, 842-848.

82 K. Guirguis, The use of nonprescription medicines among elderly patients with chronic illness and their need for pharmacist interventions, Consult Pharm 25, 2010, 433-439.

83 L.A. Hanna and C. Hughes, The influence of evidence-based medicine training on decision-making in relation to over-the-counter medicines: a qualitative study, Int J Clin Pharmacol 20, 2012, 358-366.

84 L.A. Hanna and C.M. Hughes, 'First, Do No Harm': factors that influence pharmacists making decisions about over-the-counter medication-A qualitative study in Northern Ireland, Drug Saf 33, 2010, $245-255$.

85 S. Kaae, J.M. Traulsen and L.S. Nørgaard, Customer interest in and experience with various types of pharmacy counselling - a qualitative study, Health Expect 17, 2014, 852-862.

86 C. Kippist, K. Wong, D. Bartlett and B. Saini, How do pharmacists respond to complaints of acute insomnia? A simulated patient study, Int J Clin Pharmacol 33, 2011, 237-245.

87 .S. Nielsen, J. Cameron and S. Pahoki, Opportunities and challenges: over-the-counter codeine supply from the codeine consumer's perspective, Int J Pharm Pract 21, 2013, 161-168.

88 S. Pumtong, H.F. Boardman and C.W. Anderson, A multi-method evaluation of the pharmacy first minor ailments scheme, Int J Clin Pharmacol 2011, 573-581.

89 L. Smith, T. Nguyen, C. Seeto, B. Saini and L. Brown, The role of non-clinicians in a goal setting model for the management of allergic rhinitis in community pharmacy settings, Patient Educ Couns 85, 2011, e26-e32.

90 K.A. Williams, L.M. Emmerton, R. Taylor, J. Werner and S.I. Benrimoj, Non-prescription medicines and Australian community pharmacy interventions: rates and clinical significance, Int J Pharm Pract 19, 2011, 156165.

91 T.N. Xu, A.C.D. Neto and R.J. Moles, Simulated caregivers: their feasibility in educating pharmacy staff to manage children's ailments, Int J Clin Pharmacol 34, 2012, 587-595.

92 J. Banks, A. Shaw and M.C. Weiss, The community pharmacy and discursive complexity: a qualitative study of interaction between counter assistants and customers, Health Soc Care Community 15, 2007, 313-3 